\title{
Heat shock proteins IbpA and IbpB are required for NIpl-participated cell division in Escherichia coli
}

\author{
Jing Tao' ${ }^{1}$, Yu Sang ${ }^{1}$, Qihui Teng ${ }^{1}$, Jinjing $\mathrm{Ni}^{1}$, Yi Yang ${ }^{2}$, Stephen Kwok-Wing Tsui ${ }^{2}$ and Yu-Feng Yao ${ }^{1 *}$ \\ ${ }^{1}$ Department of Microbiology and Immunology, Institutes of Medical Sciences, Shanghai Jiao Tong University School of Medicine, Shanghai, China \\ 2 School of Biomedical Sciences, The Chinese University of Hong Kong, Hong Kong, China
}

\author{
Edited by: \\ Dongsheng Zhou, Beijing Institute of \\ Microbiology and Epidemiology, \\ China \\ Reviewed by: \\ Ching-Hao Teng, National Cheng \\ Kung University, Taiwan \\ Mikael Rhen, Karolinska Institutet, \\ Sweden \\ *Correspondence: \\ Yu-Feng Yao, Department of \\ Microbiology and Immunology, \\ Institutes of Medical Sciences, \\ Shanghai Jiao Tong University \\ School of Medicine, Rm. 710, Bldg. \\ 5, 280 S. Chongqing Rd., Shanghai \\ 200025, China \\ e-mail: yfyao@sjtu.edu.cn
}

Lipoprotein NIpl of Escherichia coli is involved in the cell division, virulence, and bacterial interaction with eukaryotic host cells. To elucidate the functional mechanism of Nlpl, we examined how NIpl affects cell division and found that induction of NIpl inhibits nucleoid division and halts cell growth. Consistent with these results, the cell division protein FtsZ failed to localize at the septum but diffused in the cytosol. Elevation of NIpl expression enhanced the transcription and the outer membrane localization of the heat shock protein $\mathrm{IbpA}$ and IbpB. Deletion of either ibpA or $i b p B$ abolished the effects of NIpl induction, which could be restored by complementation. The C-terminus of $\mathrm{Nlpl}$ is critical for the enhancement in IbpA and IbpB production, and the $\mathrm{N}$-terminus of $\mathrm{NIpl}$ is required for the outer membrane localization of $\mathrm{Nlpl}$, IbpA, and IbpB. Furthermore, NIpl physically interacts with $\mathrm{IbpB}$. These results indicate that over-expression of $\mathrm{Nlpl}$ can interrupt the nucleoids division and the assembly of FtsZ at the septum, mediated by $\mathrm{lbpA} / \mathrm{lbpB}$, suggesting a role of the NIpl/lbpA/lbpB complex in the cell division.

Keywords: Nlpl, Escherichia coli, cell division, heat shock protein IbpA and IbpB, stress response

\section{INTRODUCTION}

Bacterial lipoproteins are a group of membrane proteins and play important roles in bacterial physiology and virulence, including nutrient uptake, cell division, antibiotic resistance, cell wall metabolism, transmembrane signal transduction, and adhesion to eukaryotic host cells (Crago and Koronakis, 1998; Egan et al., 2014; Nesta et al., 2014; Zuckert, 2014). Lipoproteins are synthesized as precursor forms harboring an $\mathrm{N}$-terminal signal peptide. In both Gram-negative and Gram-positive bacteria, to form mature lipoprotein, the precursors of lipoproteins are covalently modified by an amide-linked acyl group at the $\mathrm{N}$-terminal cysteine residue and subsequently removed of signal peptide. More than 90 lipoproteins have been annotated in the Escherichia coli genome and some of them have been characterized (Ichihara et al., 1981; Yu et al., 1986; Ehlert et al., 1995).

NlpI is a lipoprotein broadly distributed in Gram-negative bacteria and conserved in E. coli strains (Ohara et al., 1999). Premature NlpI is a $34-\mathrm{kDa}$ polypeptide containing 294 amino acid residues including an $\mathrm{N}$-terminal signal sequence of 18 amino acid residues. NlpI is located in the outer membrane (OM) and may be processed by Prc protease (Tadokoro et al., 2004; Teng et al., 2010). Moreover, NlpI is a typical Tetratricopeptide repeat (TPR) protein and contains five TPR motifs, which usually mediate intermolecular protein-protein interactions (Das et al., 1998).

NlpI has multiple functions. NlpI contributes to the interaction of E. coli with intestine epithelial cells and human brain microvascular endothelial cells (Barnich et al., 2004; Teng et al., 2010). NlpI facilitates the deposition of the complement regulator
C4bp to the bacterial surface to evade innate immune system (Tseng et al., 2012). Moreover, the over-production of nlpI inhibits the release of bacterial extracellular DNA (eDNA) (Sanchez-Torres et al., 2010). The homolog of NlpI inhibits biofilm formation and contributes to cell cold acclimatization in Salmonella enterica serovar Typhimurium (Rouf et al., 2011a,b).

A previous study suggested that NlpI plays a role in the bacterial cell division (Ohara et al., 1999). Insertion inactivation of $E$. coli nlpI results in abnormal cell division and formation of filaments at elevated temperature. Over-expression of nlpI in E. coli inhibits cell growth and results in the formation of ellipsoids. However, the underlying mechanism of how NlpI regulates cell division remains unknown. The first step in bacterial cytokinesis is the assembly of a stable but dynamic $Z$ ring at the site of division. FtsZ is a tubulin-like filament-forming GTPase and assembles into the $\mathrm{Z}$ ring that determines the division plane ( $\mathrm{Li}$ et al., 2013). The initial placement of FtsZ polymerization site is tightly regulated by multiple mechanisms (Wu and Errington, 2012) as are the subsequent polymer reshaping and force generation that separate the two daughter cells from each other. The interference with FtsZ polymerization disrupts the cell division (Bi and Lutkenhaus, 1993; Mukherjee et al., 1998). It is unclear whether NlpI is associated with FtsZ.

This study aims at understanding the role of NlpI in E. coli cell division. We found that the elevation of NlpI protein level not only led to severe inhibition of bacterial growth and the bacterial morphology change, but also inhibited nucleoid division and disturbed FtsZ localization in the septum in E. coli. Furthermore, we identified two small heat shock proteins (sHsps), IbpA and 
IbpB involving in the NlpI-participated cell division regulation and IbpB interacted with NlpI. Our data suggested that NlpI, IbpA, and IbpB form a complex, which most likely plays a role in nucleoid separation and FtsZ localization in cell division.

\section{MATERIALS AND METHODS BACTERIAL STRAINS AND PLASMIDS}

The bacterial strains used in this study are derivatives of $E$. coli K12 strain MG1655 or MC1000. All E. coli strains and plasmids used in this study are listed in Table 1. All primers used in this study are listed in Table S1.

\begin{tabular}{|c|c|c|}
\hline & Characteristics & Source \\
\hline \multicolumn{3}{|l|}{ STRAINS } \\
\hline MG1655 & E. coli $\mathrm{K} 12$ strain & Laboratory stock \\
\hline$\Delta n / p /$ & $\begin{array}{l}\text { The deletion mutant of } n / p / \text { in } \\
\text { MG1655 }\end{array}$ & This study \\
\hline$\triangle i b p A$ & $\begin{array}{l}\text { The deletion mutant of } i b p A \text { in } \\
\text { MG1655 }\end{array}$ & This study \\
\hline$\Delta i b p B$ & $\begin{array}{l}\text { The deletion mutant of } i b p B \text { in } \\
\text { MG1655 }\end{array}$ & This study \\
\hline$\triangle i b p A B$ & $\begin{array}{l}\text { The deletion mutant of ibpAB in } \\
\text { MG1655 }\end{array}$ & This study \\
\hline$\Delta o m p W$ & $\begin{array}{l}\text { The deletion mutant of ompW in } \\
\text { MG1655 }\end{array}$ & This study \\
\hline BTH Reporter & BacterioMatch II Validation Reporter & Stratagene \\
\hline XL1-Blue MRF' & $\begin{array}{l}\text { Host strain for propagating } \mathrm{pBT} \text { and } \\
\text { pTRG recombinants }\end{array}$ & Stratagene \\
\hline \multicolumn{3}{|l|}{ PLASMIDS } \\
\hline pQE80L & Over-expression vector & Laboratory stock \\
\hline pQE80-n/pl & $\begin{array}{l}\text { Over-expression of C-terminal } \\
\text { His-tagged protein NIpl }\end{array}$ & This study \\
\hline pQE80-n/p/-M & $\begin{array}{l}\text { Over-expression of C-terminal } \\
\text { His-tagged protein NIpl without } \\
\text { N-terminal signal sequence }\end{array}$ & This study \\
\hline pQE80-n/p/-282 & Over-expression of truncated NIpl & This study \\
\hline pQE80-n/p/-233 & Over-expression of truncated NIpl & This study \\
\hline pAC-ibpA & $\begin{array}{l}\text { ibpA with its native promoter was } \\
\text { cloned into the ECOR } \mathrm{V} \text { and } \mathrm{BamH} \text { I } \\
\text { sites of pACYC184 }\end{array}$ & This study \\
\hline pWM1410 & pBAD33-ftsZ::yfp & Ma et al., 1996 \\
\hline pRTG & $\begin{array}{l}\text { pTRG target plasmid for Bacterial } \\
\text { two-hybrid system }\end{array}$ & Stratagene \\
\hline pBT & $\begin{array}{l}\text { pBT bait plasmid for Bacterial } \\
\text { two-hybrid system }\end{array}$ & Stratagene \\
\hline pTRG-Gal11P & $\begin{array}{l}\text { Bacterial two-hybrid system control } \\
\text { plasmid }\end{array}$ & Stratagene \\
\hline pBT-LGF2 & $\begin{array}{l}\text { Bacterial two-hybrid system control } \\
\text { plasmid }\end{array}$ & Stratagene \\
\hline pBT-nlpl & pBT harboring full length of $n / p /$ & This study \\
\hline pBT-n/p/-M & $\begin{array}{l}\text { pBT harboring } n / p / \text { without } \\
\text { N-terminal signal sequence }\end{array}$ & This study \\
\hline pTRG-ibpA & pTRG harboring ibpA & This study \\
\hline pTRG-ibpB & pTRG harboring $i b p B$ & This study \\
\hline
\end{tabular}

The deletion mutants in E. coli were constructed as previously described (Datsenko and Wanner, 2000). The $\triangle i b p A$ isogenic mutant strain was replacement of $i b p A$ by the chloramphenicol resistance cassette in the $E$. coli strain MG1655. The $\triangle i b p B$, $\triangle i b p A B$ (deletion of $i b p A$ and $i b p B$ ), and $\triangle o m p W$ were constructed using the same method. $i b p A$ with its native promoter was inserted into pACYC184 vector, and the resultant plasmid pAC-ibpA was used for complementation assay. His-tagged NlpI or NlpI-M (mature NlpI without signal peptide) was expressed from $\mathrm{pQE} 80-n l p I$ or $\mathrm{pQE} 80-n l p I-\mathrm{M}$, respectively.

\section{THE GROWTH CHARACTERISTICS OF VARIOUS E. COLI STRAINS}

E. coli strain MG1655 and its derivative mutants were transformed individually with the recombinant plasmids pQE80-nlpI by calcium chloride transformation method. Overnight cultures of the strains were subcultured in $40 \mathrm{ml} \mathrm{LB}$ broth (1:100) and incubated at $37^{\circ} \mathrm{C}$ with agitation until the OD600 was 0.5 as the zero hour reading. Then the cultures were divided into two bottles. One bottle was added with $0.5 \mathrm{mM}$ isopropyl- $\beta-\mathrm{D}$ thiogalactopyranoside (IPTG) and the other one was not. The two bottles were incubated in $37^{\circ} \mathrm{C}$ while shaking at $250 \mathrm{rpm}$ for $5 \mathrm{~h}$. OD600 was monitored every hour by biophotometer (Eppendorf).

\section{MICROSCOPY MONITORING OF CELLS AND NUCLEOIDS}

Bacterial morphology was visualized by light microscopy of Gram-stained cells and scanning electron microscopy. Nucleoids were stained with $4^{\prime}, 6$-diamidino-2-phenylindole (DAPI) and observed by an Olympus fluorescence microscope according to the previously described method (Hiraga et al., 1989).

\section{MICROSCOPY MONITORING OF FtsZ-YFP}

E. coli strain MC1000 bearing FtsZ-YFP-expressing plasmid, pWM1410, was transformed with the recombinant plasmid pQE80-nlpI, pQE80-nlpI-M, pQE80-nlpI-282 or pQE80-nlpI233 , respectively. Overnight cultures of the strains were subcultured in $40 \mathrm{ml}$ LB broth (1:100), supplemented with ampicillin and chloramphenicol, and incubated at $37^{\circ} \mathrm{C}$ with agitation until the OD600 was 0.5. Then the cultures were divided into two bottles. One bottle was added with $0.5 \mathrm{mM}$ IPTG and $10 \mathrm{mM}$ $\mathrm{L}$-arabinose (Ara), the other one was added with $10 \mathrm{mM}$ Ara. The two bottles were incubated in $37^{\circ} \mathrm{C}$ while shaking at $250 \mathrm{rpm}$ for $4 \mathrm{~h}$. Bacteria were stained with DAPI. Nucleoids and FtsZ-YFP were observed by fluorescence microscope (Olympus).

\section{CELL FRACTIONATION}

Cell fractionation was carried out as described previously (Wai et al., 2003; Zhou et al., 2012). Briefly, the bacterial cells were harvested by centrifugation and washed with $10 \mathrm{mM}$ Tris- $\mathrm{HCl}$ buffer ( $\mathrm{pH}$ 8.0) followed by sonication to disrupt the cells. The cell debris and unbroken cells were removed by centrifugation at $5000 \mathrm{~g}$ for $10 \mathrm{~min}$ at $4^{\circ} \mathrm{C}$, and the supernatant was fractionated into the membrane and cytoplasmic fractions by centrifugation at $10,000 \mathrm{~g}$ for $30 \mathrm{~min}$ at $4^{\circ} \mathrm{C}$. The supernatant was cytoplasmic fraction (CP). The sediment was treated with $\mathrm{N}$-lauryl sarcosine at a final concentration of $2 \%$ at room temperature for $30 \mathrm{~min}$ and then centrifuged at $15,000 \mathrm{~g}$ for $30 \mathrm{~min}$ at $4^{\circ} \mathrm{C}$. The resulting 
sediment was OM fraction, and the supernatant was inner membrane fraction. Samples were analyzed by $15 \%$ SDS-PAGE. The discrepant bands on the 15\% SDS-PAGE were applied to mass spectrometry (MS) analysis by using ABI 4700 TOF/TOF.

\section{RNA MANIPULATION AND QUANTITATIVE REAL-TIME RT-PCR}

Total bacterial RNA was extracted using RiboPure-Bacteria kit (Ambion) and treated with DNase I to remove genomic DNA according to the manufacturer's instructions. RNA concentrations were measured using a NanoDrop spectrophotometer (Thermo). Reverse transcription (RT) was implemented using the SuperScript III First-Stand Synthesis System (Invitrogen). The quantification of the target gene mRNA level was performed by the quantitative real-time PCR (qRT-PCR) with a SYBR Premix Ex Taq II (TaKaRa) and the ABI PRISM 7500 Fast Real-Time PCR System according to the manufacturer's instructions. The primers of $n l p I, i b p A, i b p B$, omp $W$ or $16 \mathrm{~S}$ rRNA (internal control) were listed in Table $\mathrm{S} 1$.

\section{MICROARRAY ANALYSIS}

Microarray was carried out as described previously (Yao et al., 2005, 2006). A total of 7644 70-mer oligonucleotides from $E$. coli were spotted in replicate onto aminosilane slides. The oligonucleotides that are targeting backbone genes in E. coli genomes were derived from an oligonucleotide set (http://pfgrc.jcvi.org/index.php/microarray/array_description/ escherichia_coli/version $1 . h t m l)$. It is a pan E. coli genome chip and covers all the ORFs in E. coli strain MG1655. E. coli strain MG1655 harboring plasmids pQE80-nlpI or pQE80L were grown in $\mathrm{LB}$ medium at $37^{\circ} \mathrm{C}$ with agitation until the OD600 was 0.5 . Then IPTG was added at a final concentration of $0.5 \mathrm{mM}$ followed by incubation with shaking for $2 \mathrm{~h}$ at $250 \mathrm{rpm}$. Total RNA was immediately isolated by using RiboPure-Bacteria kit (Ambion) and treated with DNase I. Ten micrograms of total RNA was denatured in the presence of $600 \mathrm{ng}$ of random hexamers and $2 \mu \mathrm{l}$ of 10X dNTPs [dATP, dCTP, dGTP, and aminoallyl-dUTP (100 mM each)] (total, 20 $\mu \mathrm{l}$ ) for $5 \mathrm{~min}$ at $65^{\circ} \mathrm{C}$ and was snap-cooled on the ice. Then, cDNA synthesis was implemented by the SuperScript III First-Stand Synthesis System for RT-PCR kit (Invitrogen). Residual RNA was hydrolyzed by alkaline, and bacterial cDNA was purified by QIAquick PCR Purification Kit (Qiagen) and labeled by use of ARES Alexa Fluor dye at 488 or $594 \mathrm{~nm}$ (Molecular Probes, Invitrogen) according to the manufacturer's instructions. The labeled cDNA was purified by Centri-Spin 20 Columns (Princeton Separations). The microarray slides were prehybridized. Equal amounts of two oppositely labeled cDNA were mixed together with equal volume of SlideHybe\#3 (Ambion) and loaded onto the slide. The slide was incubated for 16 to $18 \mathrm{~h}$ at $42^{\circ} \mathrm{C}$ and washed at $55^{\circ} \mathrm{C}$ by $2 \mathrm{X}$ $\mathrm{SSC} / 0.1 \% \mathrm{SDS}, 10 \mathrm{~min}$ in $0.1 \mathrm{X}$ SSC/0.1\% SDS at room temperature twice, $5 \mathrm{~min}$ in $0.1 \mathrm{X} \mathrm{SSC}$ at room temperature twice and 2 min in 0.05X SSC. After drying, the slide was scanned at 594 and $488 \mathrm{~nm}$ with a GenePix ${ }^{\mathrm{TM}}$ 4200A Scanner (Molecular Devices). Three independent experiments were performed by reversing dyes. Image processing and data extraction were accomplished by using GenePix Pro 6.0.1.27 (Axon Instruments). Microarrays were analyzed using $\mathrm{R}$ with "limma" package. After loading the microarray data into $\mathrm{R}$, several steps were done following the limma user guides, including: Background Correction (method = "normexp," offset = 50); Within-Array Normalization (method = "loess"); Between-Array Normalization (method = "quantile"). Two kinds of plots were used to assess the normalization procedures: MA-plot (http://en.wikipedia.org/wiki/MA_plot); Individual-channel Densities.

\section{WESTERN BLOT ANALYSIS}

Protein samples were separated by SDS-PAGE and transferred to PVDF membranes. Membranes were blocked by $5 \%$ non-fat milk in Tris Buffered Saline (TBS), with $0.1 \%$ Tween-20 added. The rabbit anti-sera against NlpI (1:3000) or anti-IbpA (1:3000) or anti-Crp (1:4000), the mouse anti-sera against-His (1:4000), and the goat horseradish peroxidase (HRP)-conjugated anti-rabbit or anti-mouse IgG antibodies (1: 4000) were used as the primary or secondary antibodies to detect the target proteins. The rabbit anti-sera against NlpI is a gift from Dr. Kwang Sik Kim at Johns Hopkins University. The rabbit anti-IbpA and anti-Crp sera were customized by Beijing ComWin Biotech. The mouse antisera against-His was purchased from Tiangen. HRP-conjugated goat anti-rabbit or anti-mouse IgG antibodies were purchased from Beijing ComWin Biotech. The antibody of RNAP (RNA polymerase) was purchased from Santa Cruz and used according to manufacturer's manual. The blots were developed with Super Signal West Pico Chemiluminescent Substrate (Thermo).

\section{CO-IMMUNOPRECIPITATION (CO-IP) ASSAY}

The interactions between NlpI and IbpA or IbpB were analyzed by Co-IP according to the Pierce Crosslink Immunoprecipitation Kit (Thermo) with some modifications. E. coli strain MG1655 harboring pQE80-nlpI-M were induced with $500 \mu \mathrm{M}$ IPTG for $4 \mathrm{~h}$ and then harvested and resuspended in $1 \mathrm{ml}$ of ice cold buffer (25 mM Tris, $150 \mathrm{mM} \mathrm{NaCl,} 1$ mM EDTA, 1\% NP-40, 5\% glycerol, $\mathrm{pH}$ 7.4). After sonication, cell debris was removed by centrifugation at $10,000 \mathrm{~g}$ for $30 \mathrm{~min}$ at $4^{\circ} \mathrm{C}$. Co-IP were performed by binding of $10 \mu \mathrm{g}$ of anti-HisTag antibody to $20 \mu \mathrm{l}$ protein $\mathrm{A} / \mathrm{G}$ plus agarose for $1 \mathrm{~h}$, crosslinking, and then incubating with $150 \mu \mathrm{g}$ of cell extracts in $300 \mu \mathrm{l}$ of IP Lysis/Wash buffer for $2 \mathrm{~h}$ at $4^{\circ} \mathrm{C}$ with shaking. The beads were washed with the IP Lysis/Wash buffer. Immune complexes were eluted and analyzed by Western blot using anti-IbpA, anti-NlpI or anti-HisTag antibody.

\section{BACTERIAL TWO-HYBRID ASSAY}

The protein-protein interaction between NlpI and IbpA or IbpB was examined by BacterioMatch II Two-Hybrid System (Stratagene) according to the previous description (Du et al., 2012). The $n l p I-M$ and $n l p I$ were cloned into the pBT individually. The $i b p A$ and $i b p B$ genes were cloned into the pTRG individually. The reporter strain was co-transformed with the recombinant plasmids $\mathrm{pBT}-n l p I-\mathrm{M}$ and pTRG-ibpA or pTRG-ibpB and streaked onto the dual selective screening medium (DSSM) containing $5 \mathrm{mM} \mathrm{3-amino-1,2,4-triazole}$ (3-AT), $12.5 \mathrm{mM}$ streptomycin, $12.5 \mathrm{mM}$ tetracycline, $25 \mathrm{mM}$ chloramphenicol and $50 \mathrm{mg} / \mathrm{ml}$ kanamycin. A cotransformant containing pBT-LGF2 and pTRG-Gal11P was used as a positive control for expected growth on DSSM. A cotransformant 
containing pBT and pTRG was used as a negative control. Bacterial two-hybrid assay between the recombinant plasmids pBT-nlpI and pTRG-ibpA or pTRG-ibpB were carried out similarly.

\section{STATISTICAL ANALYSIS}

qRT-PCR data are shown as means \pm standard deviations. Statistical analyses were performed using GraphPad Prism 5. Paired $t$-tests were used to determine $P$-values. With regard to the microarray data analysis, after analyzed by $\mathrm{R}$ package, genes with the expression fold change greater than 2 or less than 0.5 and $P$-value $<0.01$ were considered as differential expression.

\section{RESULTS}

\section{INHIBITION OF E. COLI GROWTH BY INDUCTION OF $\boldsymbol{n I p I}$}

The previous study found that the over-expression of $n l p I$ affects cell growth and cellular morphology in E. coli strain MO101 (Ohara et al., 1999). It promoted us to test whether this phenotype is unique in a certain E. coli strain or not. Therefore, E. coli strain MG1655 harboring plasmid pQE80-nlpI was tested. When the bacteria were induced with $0.5 \mathrm{mM}$ IPTG at $37^{\circ} \mathrm{C}$, the cell growth was inhibited severely (Figure 1A). Light microscopy showed the increase of width and length of cell and the aggregation of bacterial cells after induction (Figure 1B), and scanning electron microscopy illustrated the appearance of swollen prolate ellipsoids and cell envelope invagination and damage (Figure 1C). Since the bacterial filamentation was observed with over-expression of $n l p I$, we want to check the nucleoids division in these cells. DAPI staining showed that the bacterial nucleoids were anomalous after the induction of $n l p I$ compared with control cells (Figure 1D). We suspect that over-expression of $n l p I$ affected the cell division by influencing nucleoids division. Another E. coli strain DH5 $\alpha$ was applied to the above assays and showed similar phenotypes (data not shown). This means that the phenotypes we observed are not strain specific.

During export of OM lipoprotein across the cytoplasmic membrane, the lipoprotein signal peptide is cleaved by signal peptidase, which is critical for the function of lipoprotein (Zuckert, 2014). In order to investigate the role of NlpI signal peptide in bacterial growth and division, we constructed pQE80-nlpI-M that contained the sequence in accordance with the mature polypeptide (residues 20-294, lacking the signal sequence and Cys19) (Figure 1E). With the IPTG induction, the growth curve showed the bacterial growth rate was comparable to that of control strain

\section{A}

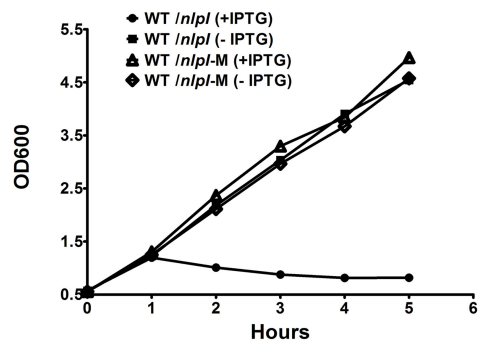

B

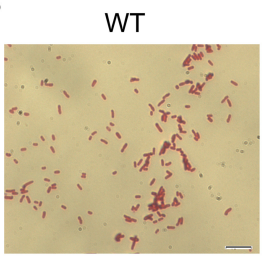

C

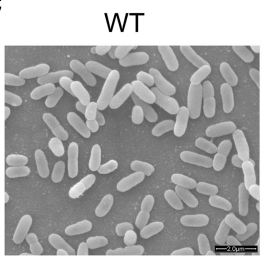

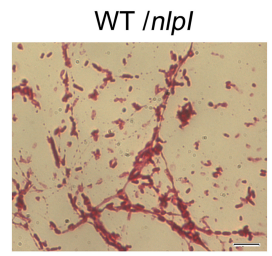

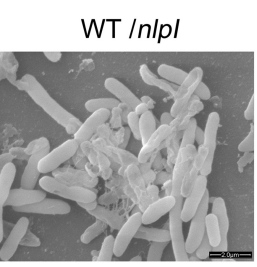

D

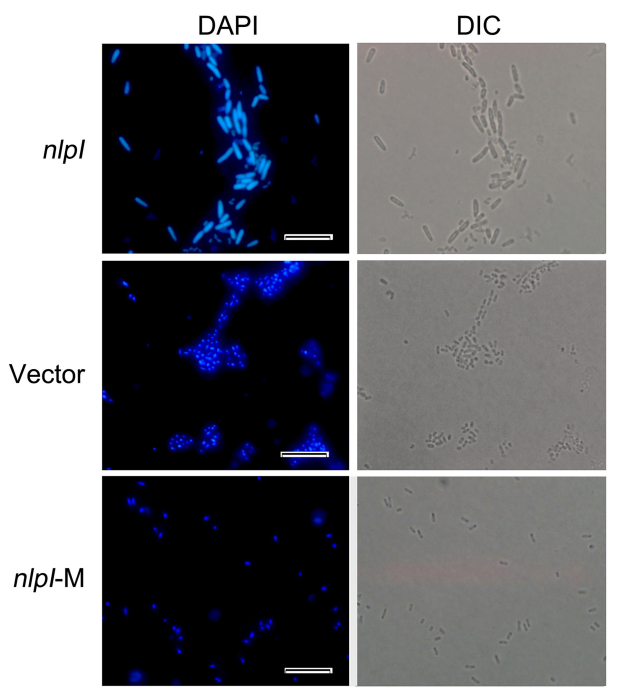

E

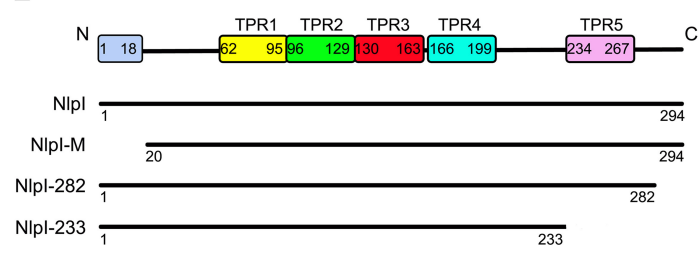

FIGURE 1 | The elevation of $n / p l$ in $E$. coli inhibited the host cell growth. (A) The growth curves of the wild type (WT) strain MG1655 harboring the plasmid pQE80-n/p/ or pQE80-n/p/-M in the presence or absence of inducer. Results shown are representative of three independent experiments. (B) The cellular morphology of Gram-stained bacteria. E. coli strain MG1655 harboring plasmid pQE80-n/p/ was incubated in LB broth at $37^{\circ} \mathrm{C}$ with shaking until OD600 reached 0.5 . Then the bacteria were induced by adding IPTG to a final concentration of $0.5 \mathrm{mM}$ for $4 \mathrm{~h}$. The cells were subsequently stained with Gram stain and observed by light microscope. Magnification, X1000. (C) Scanning electron microscopy examined the cellular morphology change and aggregation. Magnification, X10,000. (D) Bacteria were stained with DAPI and nucleoids were observed by fluorescence microscopy. Magnification, X1000. Scale bar, $10 \mu \mathrm{m}$. (E) Schematic presentation of Nlpl and its three variants. The full length NIpl contains 294 amino acids including an N-terminal signal sequence and five TPR motifs. Nlpl-M represents a mature NIpl protein without $\mathrm{N}$-terminal signal sequence. NIpl-282 and NIpl-233 are truncated Nipl lacking the C-terminal 12 residues and the fifth TPR, respectively. 
without induction (Figure 1A). DAPI staining showed that the bacterial nucleoids were normal (Figure 1D). This highly suggests that the signal peptide sequence is required for the phenotypes caused by NlpI over-expression.

It has been shown that the C-terminus of NlpI is critical to correct the thermosensitivity of the $n l p I$ mutant (Tadokoro et al., 2004). Moreover, the amino acid sequence showed that NlpI contains five TPR motifs (Wilson et al., 2005). To determine the role of NlpI C-terminus, we constructed two plasmids, pQE80-nlpI282 (lacking the C-terminal 12 residues) and pQE80-nlpI-233 (lacking the C-terminal 61 residues including the fifth TPR) (Figure 1E). These two plasmids were introduced individually into E. coli strain MG1655 followed by induction with IPTG. We found that the over-expression of $n l p I-282$ severely inhibited the bacterial growth, but $n l p I-233$ failed (data not shown). DAPI staining showed the bacterial nucleoids in the over-expression of $n l p I-282$ cell were anomalous, which was similar to that of the full length $n l p I$ (Figure 2), while the nucleoids were normally divided in the nlpI-233 over expression cells (Supplementary Figure S1). The above experiments suggested that NlpI was involved in

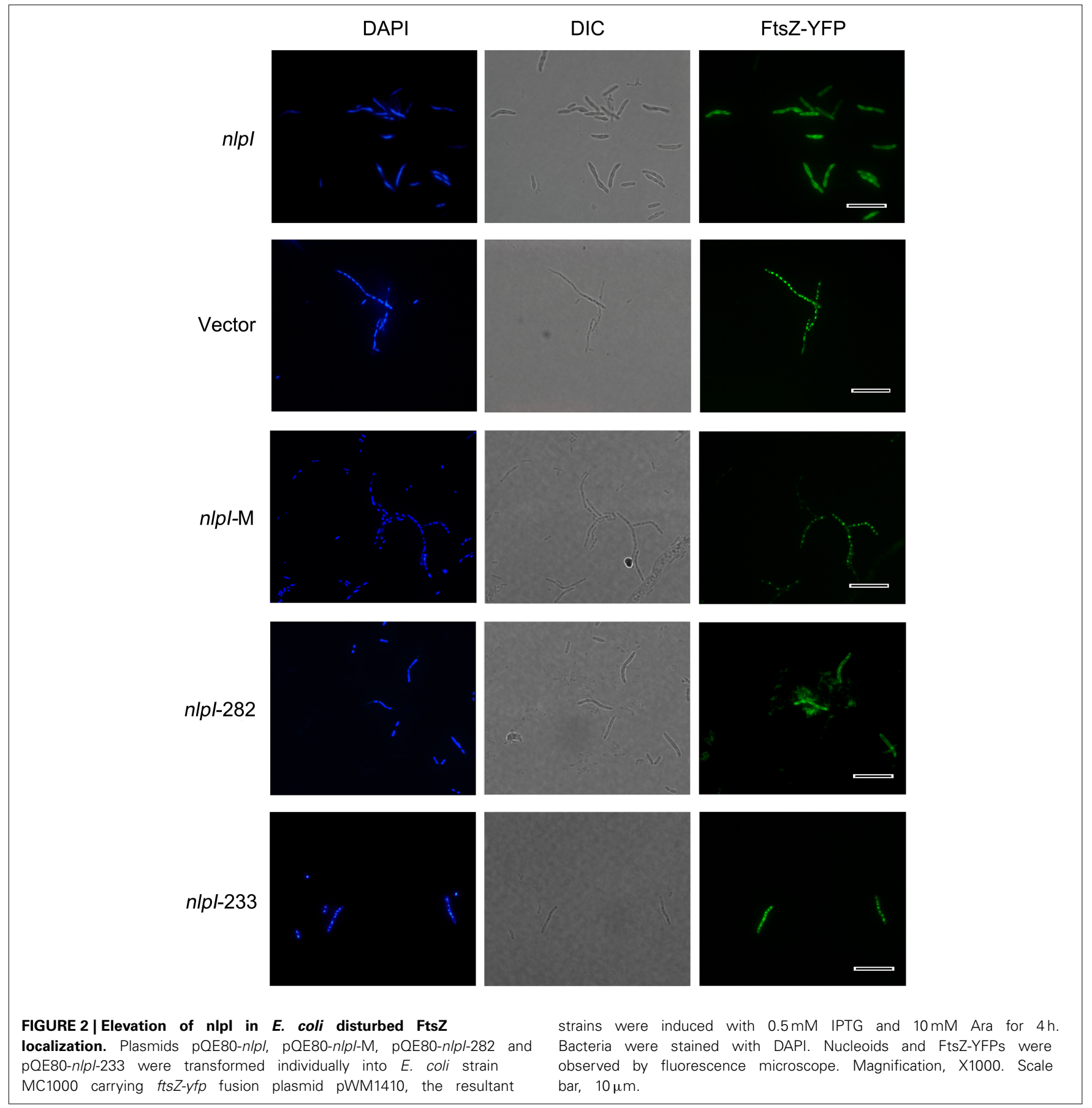


the bacterial division in E. coli, and both N-terminal and Cterminal sequences were critical to the role of NlpI in the cell division.

\section{ELEVATION OF $\boldsymbol{n l p I}$ PROTEIN LEVEL DISTURBED FtsZ LOCALIZATION}

Bacterial cell division requires accurate localization of the cytoskeletal protein FtsZ at the nascent division site and assembly into Z-ring (Bi and Lutkenhaus, 1991). Since over-expression of $n l p I$ inhibited nucleoids division in E. coli, we speculate that the increase of NlpI protein level perhaps influences FtsZ localization. We induced $n l p I$ and $f t s Z-y f p$ with IPTG and arabinose, respectively in E. coli strain MC1000, and found that the nucleoids were anomalous and FtsZ-YFPs were diffusive in the cytoplasm in nlpI over-expression cells. Induction of $n l p I-282$ and ftsZ- $y f p$ caused the same change of the nucleoids and FtsZ localization as that of the full length of nlpI (Figure 2). However, both nucleoid division and FtsZ localization were normal after induction of $n l p I-\mathrm{M}$ or $n l p I-233$ (Figure 2). These results indicated that increase of NlpI inhibited nucleoid division and interfered with FtsZ localization, and both $\mathrm{N}$-terminus and $\mathrm{C}$-terminus contributed to this process.

\section{THE TRANSCRIPTOME WITH ELEVATION OF nIpI PROTEIN LEVEL}

The above results suggest that NlpI is involved in the nucleoid separation and FtsZ localization. Since we failed to detect the interaction of NlpI and FtsZ in Co-IP assay (data not shown), we performed microarray analyses to identify other factors that may participate in this process. The total RNA from E. coli strain MG1655 harboring plasmid pQE80-nlpI or pQE80L induced with IPTG were isolated followed by reverse transcription, fluorescent labeling, hybridization, microarray scanning, and data were analyzed as previous description (Yao et al., 2005, 2006).

A total of 142 genes (68 up-regulated and 74 down-regulated genes) were found to be differentially expressed (Supplementary Tables S2 and S3). Some up-regulated and down-regulated genes were listed in Table 2 . We did not find the change of transcriptional level of $f t s Z$, but we found that the transcriptional level of genes encoding heat shock proteins and chaperones, such as $i b p A, i b p B$, groES, groEL, htpG, clpB, dnaK, were significantly increased under NlpI overexpression. The expression of genes encoding outer membrane proteins, such as ompW and slp was significantly decreased. Small heat shock proteins lbpA and lbpB (inclusion body-associated protein) protect heat-denatured proteins from irreversible aggregation (Kuczynska-Wisnik et al., 2004). The heat shock proteins and chaperones including GroELGroES, DnaK, and ClpB are involved in preventing aggregation of heat-denatured proteins (Mogk et al., 2002; Sorensen and Mortensen, 2005). We suspect that the significantly up-regulated expression of above genes may be a concomitant event of the elevation of NlpI protein level and play an important role in the NlpI-participated cell division. Quantitative RT-PCR (qRTPCR) showed that the level of $i b p A$ mRNA was increased by $14,839.7 \pm 3672.3$ fold (means \pm standard deviations), and that the levels of $i b p B$ mRNA was obviously increased by $9947.3 \pm$ 2867.9 fold in the NlpI over-expression cells (Figures 3A,B),
Table 2 | Genes with differential expression after the elevation of $\boldsymbol{n} / \boldsymbol{p l}$.

\begin{tabular}{|c|c|c|c|}
\hline Annotation & Gene & LogFC & $P$-value \\
\hline Heat shock protein & $i b p A$ & 7.25 & $0.00 \mathrm{E}+00$ \\
\hline Heat shock protein & $i b p B$ & 7.04 & $0.00 \mathrm{E}+00$ \\
\hline Dihydrolipoamide dehydrogenase & IpdA & 4.00 & $0.00 \mathrm{E}+00$ \\
\hline Co-chaperonin & groES & 3.96 & $0.00 \mathrm{E}+00$ \\
\hline Heat shock protein 90 & $h t p G$ & 3.69 & $0.00 \mathrm{E}+00$ \\
\hline Chaperonin & groEL & 3.68 & $1.00 \mathrm{E}-02$ \\
\hline Cold shock protein & $\operatorname{csp} E$ & 3.44 & $1.00 \mathrm{E}-02$ \\
\hline D-ribose transporter subunit & $r b s B$ & 3.23 & $0.00 \mathrm{E}+00$ \\
\hline DNA-binding transcriptional activator & marA & 3.05 & $0.00 \mathrm{E}+00$ \\
\hline Protein disaggregation chaperone & $c / p B$ & 2.57 & $0.00 E+00$ \\
\hline Alpha-ketoglutarate transporter & kgtP & 2.53 & $0.00 \mathrm{E}+00$ \\
\hline Oligopeptide ABC transporter & oppA & 2.53 & $0.00 \mathrm{E}+00$ \\
\hline Molecular chaperone & $d n a K$ & 2.36 & $0.00 \mathrm{E}+00$ \\
\hline $\begin{array}{l}\text { Galactitol-specific PTS system component } \\
\text { IIB }\end{array}$ & gatB & 2.35 & $0.00 E+00$ \\
\hline $\begin{array}{l}\text { ATP-dependent protease ATP-binding } \\
\text { subunit }\end{array}$ & $h s / U$ & 2.32 & $0.00 \mathrm{E}+00$ \\
\hline $\begin{array}{l}\text { Trehalose(maltose)-specific PTS system } \\
\text { components IIBC }\end{array}$ & $\operatorname{tre} B$ & 2.14 & $0.00 E+00$ \\
\hline Universal stress protein & uspA & 2.03 & $0.00 \mathrm{E}+00$ \\
\hline RNA polymerase sigma factor & rpos & 2.01 & $0.00 \mathrm{E}+00$ \\
\hline Biofilm formation regulatory protein & bssS & 1.94 & $1.00 \mathrm{E}-02$ \\
\hline $\begin{array}{l}\text { ATP-dependent Clp protease proteolytic } \\
\text { subunit }\end{array}$ & $c / p P$ & 1.89 & $1.00 E-02$ \\
\hline Heat shock protein & $h t p X$ & 1.88 & $0.00 \mathrm{E}+00$ \\
\hline Chaperone protein & dnaJ & 1.66 & $0.00 \mathrm{E}+00$ \\
\hline Thiamine transporter membrane protein & thiP & 1.27 & $0.00 \mathrm{E}+00$ \\
\hline Anti-sigma 28 factor FlgM & flgM & -2.05 & $3.96 \mathrm{E}-03$ \\
\hline Outer membrane protein $W$ & ompW & -1.91 & $9.83 E-04$ \\
\hline Outer membrane protein & slp & -1.80 & $8.73 E-04$ \\
\hline $\begin{array}{l}\text { Cytochrome d terminal oxidase, } \\
\text { polypeptide subunit I }\end{array}$ & cydA & -1.64 & $1.22 \mathrm{E}-03$ \\
\hline Melibiose:sodium symporter & melB & -1.38 & $5.16 \mathrm{E}-03$ \\
\hline $\begin{array}{l}\text { Glutamine } A B C \text { transporter periplasmic } \\
\text { protein }\end{array}$ & $g \ln H$ & -1.32 & 4.36E-03 \\
\hline F0F1 ATP synthase subunit beta & $\operatorname{atp} D$ & -1.27 & $3.15 E-03$ \\
\hline Methyl-accepting chemotaxis protein III & $\operatorname{trg}$ & -1.23 & 7.49E-03 \\
\hline $\begin{array}{l}\text { Glucose-1-phosphatase/inositol } \\
\text { phosphatase }\end{array}$ & agp & -1.19 & $3.99 E-03$ \\
\hline $\begin{array}{l}\text { Anaerobic glycerol-3-phosphate } \\
\text { dehydrogenase subunit B }\end{array}$ & $g / p B$ & -1.14 & $6.57 \mathrm{E}-03$ \\
\hline $\begin{array}{l}\text { Citrate reductase cytochrome c-type } \\
\text { subunit }\end{array}$ & napB & -1.14 & $6.80 E-03$ \\
\hline Ammonium transporter & $a m t B$ & -1.13 & $9.61 \mathrm{E}-03$ \\
\hline Chemotaxis regulatory protein $\mathrm{CheY}$ & cheY & -1.13 & $6.08 \mathrm{E}-03$ \\
\hline Maltose transporter permease & malG & -1.11 & $5.05 E-03$ \\
\hline $\begin{array}{l}\text { Anaerobic dimethyl sulfoxide reductase } \\
\text { subunit B }\end{array}$ & $d m s B$ & -1.06 & $7.99 \mathrm{E}-03$ \\
\hline Undecaprenyldiphospho- & murG & -1.05 & $5.45 \mathrm{E}-03$ \\
\hline
\end{tabular}

muramoylpentapeptide

beta-N-acetylglucosaminyltransferase

LogFC, the log2-fold-change. 

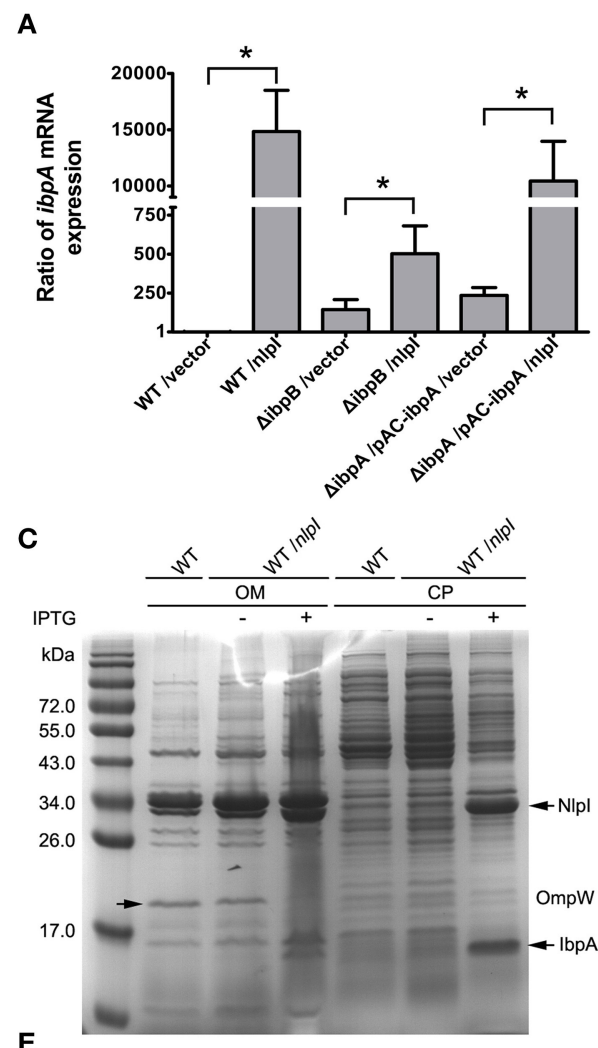

E

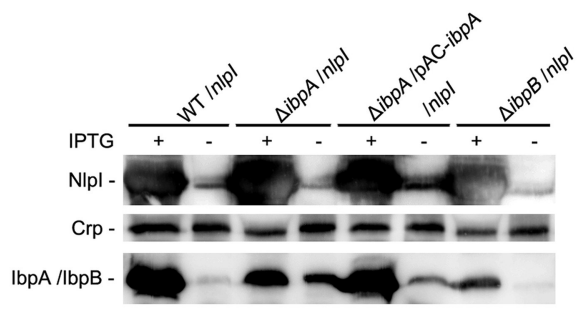

FIGURE 3 | IbpA and IbpB are required for the NIpl-participated cell division. (A) The levels of ibpA mRNA were significantly increased with induction of $n / p /$ in wild type strain or complementation strain. The level of ibpA mRNA in wild type strain with vector was set at one. The levels of ibpA mRNA in MG1655/pQE80-n/p/ and $\triangle i b p B / p Q E 80-n / p /$ were increased $14,839.7 \pm 3672.3$ and $502.2 \pm 178.8$ fold, respectively, compared with control. The level of ibpA mRNA in $\triangle i b p A / p A C-i b p A / p Q E 80-n / p /$ was increased by $10,437.3 \pm 3536.5$ fold. The error bars indicated the SD representing the means from three independent experiments. (B) The levels of ibpB mRNA were the remarkably increased with over-expression of $n / p /$ in wild type strain. The level of $i b p B$ mRNA of wild type strain with empty vector was set at 1 . The levels of ibpB mRNA of wild type strain and $\triangle i b p A$ with

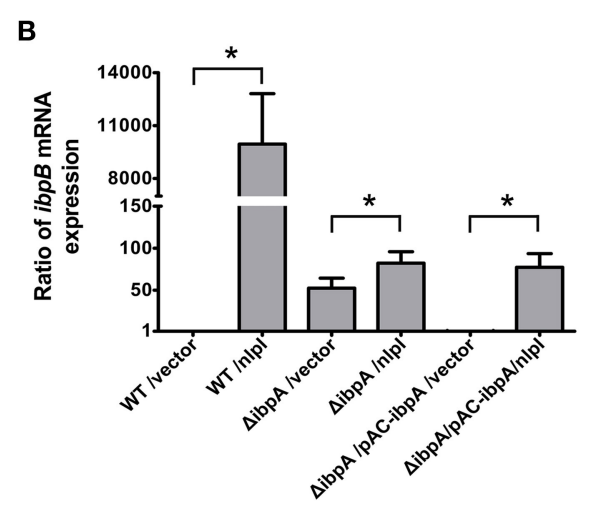

D

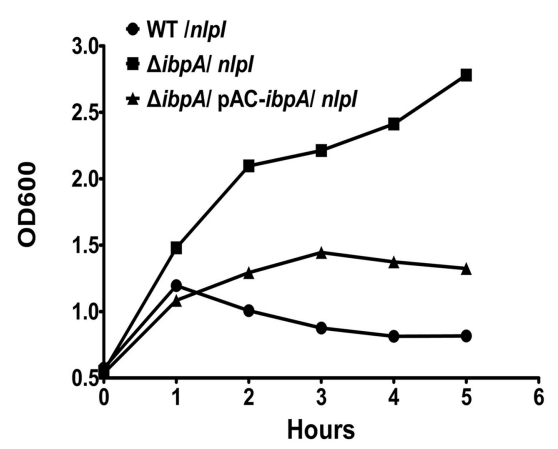

$\mathbf{F}$

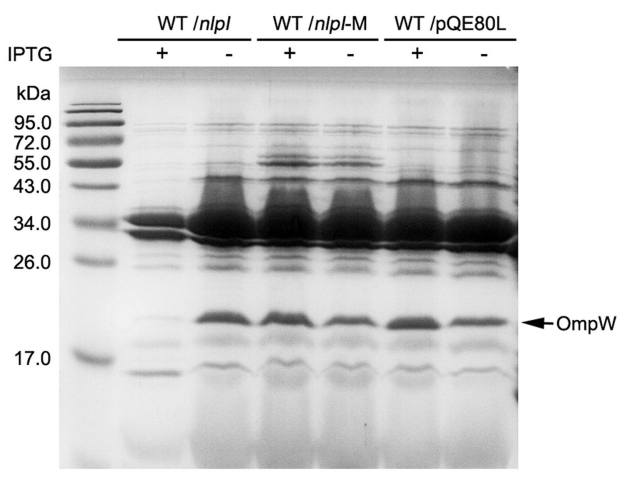

inducer were increased by $9947.3 \pm 2867.9$ and $82.2 \pm 13.6$ fold, respectively. The level of $i b p B$ mRNA in $\triangle i b p A / p A C$-ibpA was increased by $77.2 \pm 16.3$ fold (C) The induction of $n / p /$ caused the dramatic change of protein profiles of CP and $\mathrm{OM}$ of the host cells. Mass spectrometry identified two significantly discrepant bands, IbpA and OmpW. (D) The growth curves of wild type strain, $\triangle i b p A$ and the complementation strain of $\triangle i b p A$ with over-expression of $n / p /$ were determined. The growth of wild type strain and the complementation strain of $\triangle i b p A$ were inhibited. (E) Western blot analysis of the protein levels of $\mathrm{NIpl}$ or IbpA/lbpB in CP of the host cells, using polyclonal antibodies anti-NIpl, anti-Crp and anti-IbpA. Crp is a cytoplasmic protein marker. (F) The OM protein profiling of MG1655/pQE80-n/p/, MG1655/pQE80-n/p/-M and MG1655/pQE80L with/without inducer. $* P<0.05$. and the transcriptional level of ompW was decreased dramatically (Supplementary Figure S2A). These findings confirmed the result of microarray (Table 2 ), and illustrated that the transcription of $i b p A$ and $i b p B$ was activated by over-expression of $n l p I$.

\section{ibpA AND ibpB ARE REQUIRED FOR THE $n$ Ipl-PARTICIPATED CELL DIVISION}

Since the over expression of $n l p I$ cause the expression change of genes, such as $i b p A, i b p B$ and $o m p W$, we speculate that the protein profiling in the $n l p I$-induced cells differs from that of control 
cells. The bacterial cells were separated into two fractions: CP and OM. SDS-PAGE analysis showed that the over-expression of nlpI caused the dramatic changes of the proteins profiling in $\mathrm{CP}$ and $\mathrm{OM}$ of the host cells compared with those of the control cells (Figure 3C). The two significantly discrepant bands on the SDS-PAGE were applied to MS analysis. One band with increased amount was identified as the heat shock protein IbpA, while the other decreased band was outer membrane protein OmpW (Supplementary Table S4). These findings confirmed the result of microarray and qRT-PCR, and indicated that the elevation of NlpI protein level affected bacterial transcriptome and proteome.

Since the expression of $i b p A$ and $i b p B$ are significantly elevated in NlpI over expression cells, we sought to unveil their contribution in NlpI-participated cell division. The $i b p A$ and $i b p B$ form an operon, and are regulated by the $\sigma^{32}$ protein, which is encoded by $r p o H$. The open reading frames are separated by $111 \mathrm{bp}$. A $\sigma^{54}$-dependent promoter locates upstream of $i b p B$ (Allen et al., 1992; Kuczynska-Wisnik et al., 2001). IbpA and IbpB appear in aggregated protein fractions after heat shock (Laskowska et al., 1996). To test whether IbpA and IbpB are involved in the NlpIparticipated cell division, we constructed the deletion mutants of $i b p A, i b p B$, and $i b p A B(i b p A$ and $i b p B)$ in $E$. coli, and induced $n l p I$ in these mutants. Surprisingly, the growth curves showed that the elevation of NlpI did not inhibit cell growth of these three deletion mutants (Figure 3D, Supplementary Figure S2B). After complementation of a copy of $i b p A$ with its native promoter into the deletion mutant of $i b p A$, we found that over-expression of nlpI could inhibit the cell growth (Figure 3D). qRT-PCR showed that the level of $i b p A$ mRNA in the complementation strain was restored to the level of the wild type strain (Figure 3A). The inhibition of cell growth by the over-expression of $n l p I$ was abolished when $i b p B$ or $i b p A B$ was deleted (Supplementary Figure $\mathrm{S} 2 \mathrm{~B})$. Interestingly, qRT-PCR results showed that the level of $i b p A$ mRNA in $\triangle i b p B$ was increased by $502.2 \pm 178.8$ fold after the $n l p I$ induction, but was significantly lower than in MG1655 or the complementation strains of $\triangle i b p A$ (Figure 3A, Supplementary Figure S2C). Likewise, we detected that the level of $i b p B$ mRNA in $\triangle i b p A$ was increased by $82.2 \pm 13.6$ fold after the $n l p I$ overexpression, which was strikingly lower than that of the wild type strain with IPTG induction (Figure 3B). These results indicated that both IbpA and IbpB were required for the NlpI-participated cell division, and IbpA promoted the transcription level of $i b p B$ in the over-expression of nlpI cells, and vice versa.

qRT-PCR showed that the transcriptional level of $i b p B$ was increased by $52.4 \pm 11.9$ fold in $\triangle i b p A$ cells compared with wild type strain, which could be restored by trans-complementation (Figure 3B). We prepared the polyclonal antibody of IbpA and NlpI and tested the expression of NlpI and IbpA/IbpB in the cytoplasm by Western blot. Since IbpA and IbpB, whose molecular masses were both about $16-\mathrm{kDa}$, shared $48 \%$ identity at the amino acid level, the IbpA polyclonal antibody can recognize both IbpA and IbpB. We found the result of Western blot was consistent with that of qRT-PCR (Figure 3E). Western blot detected IbpB in $\triangle i b p A$ cells and IbpA in the complementation strain of $\triangle i b p A$ (Figure 3E). These results showed that IbpA inhibited transcription of $i b p B$ in wild type strain in accord with the previous finding (Gaubig et al., 2011). The level of $i b p A$ mRNA were increased by $144.2 \pm 64.4$ fold in $\triangle i b p B$ cells compared with that of wild type strain (Figure 3A). This means that IbpB inhibited the transcription of $i b p A$.

With the elevation of NlpI, DAPI staining showed that the nucleoids divided normally in both $\triangle i b p A$ and $\triangle i b p B$, but the nucleoids were anomalous in the complementation strains of $\triangle i b p A$ (Supplementary Figure S2D). Furthermore, the wild type strain MG1655 with either over-expression of $i b p A$ or $i b p B$, or both $i b p A$ and $i b p B$, showed normal growth and nucleoids division (Supplementary Figure S2D). Therefore, these data indicated that nucleoids division defect cause by NlpI overexpression was dependent on IbpA and IbpB in E. coli, and other factor(s) may be involved in this process.

To further test whether the decrease of OmpW is related to the NlpI-participated cell division, we constructed the deletion mutant of ompW followed by the over-expression of $n l p I$. The growth of $\Delta o m p W$ was inhibited as the wild type strain when $n l p I$ was induced (Supplementary Figure S2B). We found both protein and mRNA levels of ompW were decreased by the over-expression of $n l p I$ in the deletion mutants of $i b p A$, $i b p B, i b p A B$ and $i b p A$ complementation strains (Supplementary Figures S2A,E). Interestingly, SDS-PAGE showed that OmpW was not decreased by increasing NlpI-M protein level (Figure 3F). These results indicated that the over-expression of full length $n l p I$ inhibited the expression of ompW in an IbpA/B independent manner.

\section{C-TERMINUS OF nIpl INVOLVED IN THE TRANSCRIPTION OF ibpA AND ibpB}

It has been shown that the C-terminus of NlpI is critical to its function (Tadokoro et al., 2004), we want to test the role of the C-terminal NlpI in the transcription of $i b p A$ and $i b p B$ by overexpressing of $n l p I-282$ or $n l p I-233$ in the wild type strain. Western blot showed that the amount of IbpA/IbpB was increased in CP fraction in the over-expression of $n l p I-282$ cells, but not in the over-expression of $n l p I-233$ cells (Figure 4A). qRT-PCR showed that the levels of $i b p A$ and $i b p B$ mRNA were not elevated in the over-expression of $n l p I-233$ cell, but increased by $511.8 \pm 4.4$ and $1090.7 \pm 81.4$ fold after the induction of $n l p I-282$, respectively (Figures 4B,C, Supplementary Figure S3). These results indicated that $\mathrm{C}$-terminus of NlpI played an important role in the transcription of $i b p A$ or $i b p B$.

\section{$n$ IpI MEDIATED IbpA AND IbpB LOCALIZATION TO OUTER MEMBRANE}

It has been shown that $\mathrm{IbpA}$ and $\mathrm{IbpB}$ are localized to $\mathrm{OM}$ (Laskowska et al., 1996), and our data showed that overexpression of $n l p I$ promoted the expression of $i b p A$ and $i b p B$, we speculate that the localization of IbpA and IbpB might be affected. To test this hypothesis, we over-expressed $n l p I$ or $n l p I$ $\mathrm{M}$ in the wild type cells followed by RNA isolation and cell fractionation. qRT-PCR showed that the mRNA levels of $i b p A$ and $i b p B$ were increased after the over-expression of $n l p I$ or $n l p I-M$ (Figures 5A,B). We detected the dramatic increase of NlpI in the OM of cells with over-expression of $n l p I$ by using His-tag antibody, but not in the cells with over-expression of nlpI-M (Figure 5D). This result showed that N-terminal signal sequence is required for NlpI localization to OM. The amount of 

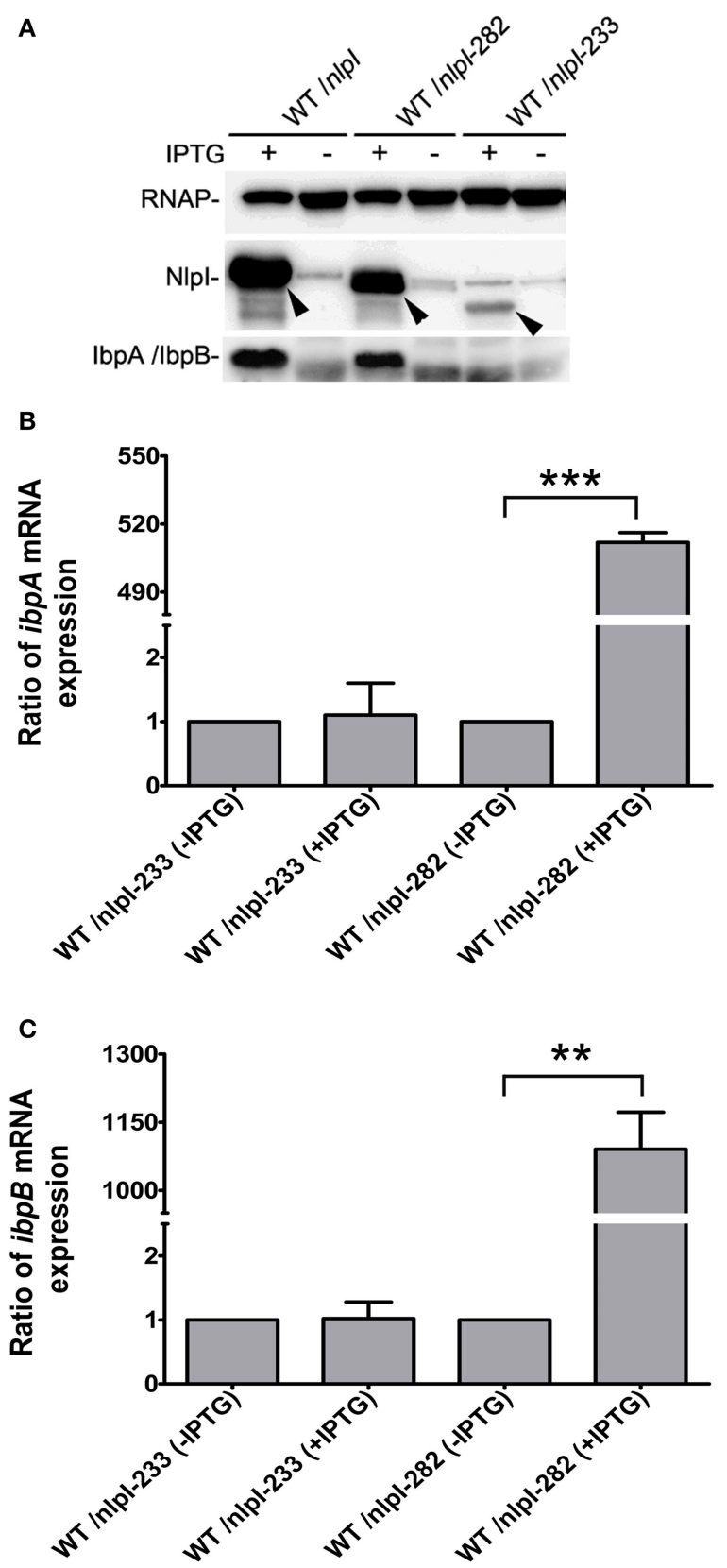

FIGURE 4 | C-terminal of NIpl involved in the transcription of $i b p A$ and ibpB. (A) Western blot with anti-NIpl and anti-lbpA antibody detected that $\mathrm{IbpA}$ and $\mathrm{IbpB}$ were increased in CP after the induction of $n / p /$ or $n / p /-282$, but not in the over-expression of $n / p /-233$ cells. (B, C) qRT-PCR showed that the mRNA levels of $i b p A$ and $i b p B$ after the induction of n/pl-282 were increased by $511.8 \pm 4.4,1090.7 \pm 81.4$ fold, respectively, compared with corresponding stain without IPTG. But the mRNA levels of ibpA and ibpB after the induction of $n / p /-233$ were $1.1 \pm 0.5,1.02 \pm 0.26$ fold, respectively, compared with corresponding stain without IPTG. ${ }^{* * *} P<0.001$, ** $P<0.01$

IbpA/IbpB was increased in CP fraction after the over-expression of either $n l p I$ or $n l p I-M$ as expected (Figures 5C,E). However, the amount of IbpA/IbpB in OM fraction was increased only after the over-expression of $n l p I$, but not $n l p I-\mathrm{M}$ (Figure 5D). These data indicated that localization of $\mathrm{NlpI}$ to $\mathrm{OM}$ is related to the process of IbpA and IbpB localizing to OM.

\section{NIpI PHYSICALLY INTERACTS WITH IbpB}

Upregulation of IbpA and IbpB by NlpI over-expression and their co-localization to the OM suggested that these two proteins may function together with NlpI to cause growth retardation. Thus, we checked the interaction between NlpI and IbpA or IbpB. Coimmunoprecipitation (Co-IP) assay was applied to detect the potential interaction between NlpI and IbpA/IbpB. Co-IP assay was performed by binding of His-tag antibody to protein $A / G$ plus agarose beads and incubating with cell extracts. Immune complexes were eluted in elution buffer and analyzed by Western blot using anti-IbpA and anti-HisTag antibody (Figure 6A). The results showed that NlpI interacted with IbpA and/or IbpB. We expressed and purified NlpI-M in wild type strain, $\triangle i b p A$, $\triangle i b p B$ or $\triangle i b p A B$, respectively. Western blot and MS analysis showed IbpA and IbpB were co-eluted when NlpI-M was purified in wild type strain (Supplementary Figure S4 and Table S5). Moreover, Western blot confirmed that IbpB was co-eluted in purified NlpI-M from $\triangle i b p A$. We used another method, bacterial two-hybrid assay to confirm the interaction between NlpI and IbpA/IbpB. As shown in Figures 6B,C, the co-transformants containing IbpB and NlpI or NlpI-M grew well-on DSSM. But the co-transformants containing IbpA and NlpI or NlpI-M had no obvious growth phenomenon on DSSM (data no shown). These results suggested that NlpI, IbpA, and IbpB may form a complex to mediate the cell division.

\section{DISCUSSION}

In this study, we found that the over-expression of NlpI severely inhibited the bacterial growth, influenced nucleoids division and FtsZ localization in the septum, and that nlpI-282 lacking Cterminal 12 amino acid residues sequence showed similar phenotypes. However, the over-expression of $n l p I-M$ and $n l p I-233$ failed to inhibit nucleoids division and FtsZ localization. Considering the fact that we did not detect the interaction between NlpI and FtsZ, we speculated that NlpI participated in cell division by inhibiting nucleoid division and interfering with FtsZ localization in a contact independent manner.

The previous study showed that the over-expression of recombinant proteins result in heat shock-like response in E. coli (Gill et al., 2000). Under this condition, the mRNA levels of $i b p A$ and $i b p B$ are highly expressed (Gill et al., 2000; Jurgen et al., 2000). IbpA and IbpB are molecular chaperone proteins (Jiao et al., 2005; Strozecka et al., 2012), which are involved in resistance to heat and superoxide stresses (Kitagawa et al., 2000) and protect enzymes from inactivation by heat (Kitagawa et al., 2002). IbpA decreases the size of substrate complexes and inhibits their further processing (Ratajczak et al., 2009). IbpB, which is associated with its substrate via forming complexes with IbpA (Matuszewska et al., 2005), facilitates substrate transfer to the Hsp70/40 and the Hsp100 chaperone machinery (Ratajczak et al., 2009). In this study, the DNA microarray analysis showed that the expression of IbpA, IbpB, the Hsp70 chaperone DnaK and the Hsp100 chaperone ClpB significantly increased after the over-expression of nlpI (Table 2). Furthermore, the protein amount of IbpA and 


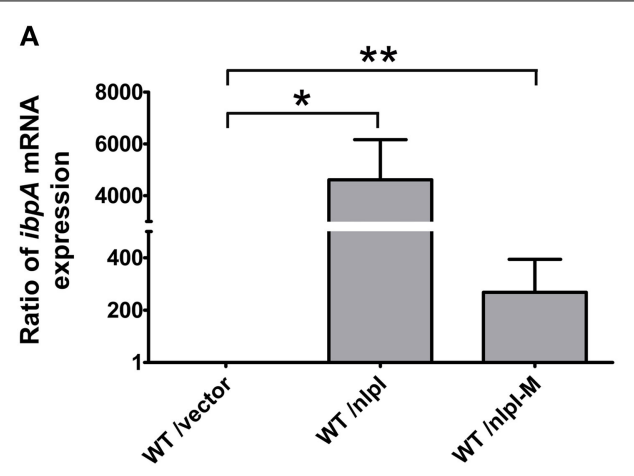

。

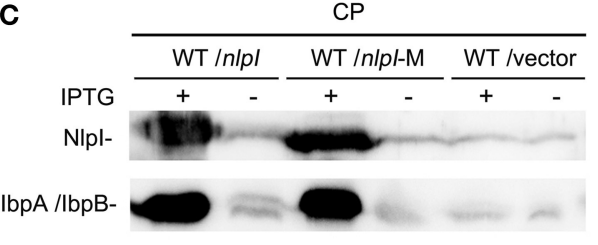

$\mathbf{E}$

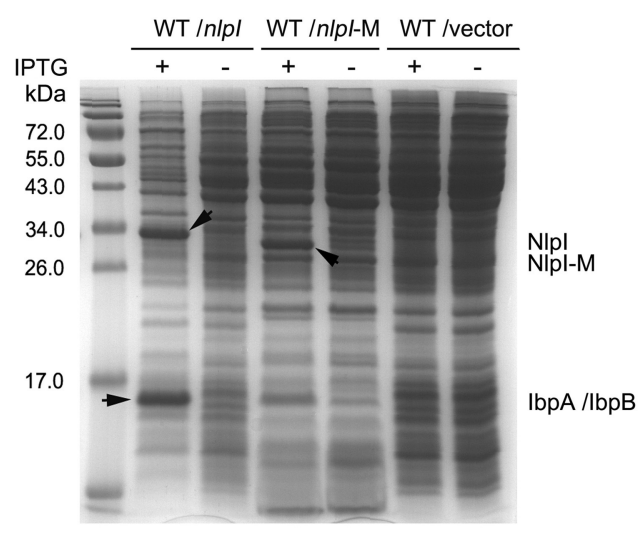

FIGURE 5 | NIpl mediated IbpA and lbpB localization to outer membrane. (A, B) qRT-PCR showed that the mRNA levels of $i b p A(\mathbf{A})$ and $i b p B(\mathbf{B})$ by over-expression of $n / p /$ or $n / p /-M$ were increased dramatically, compared with control. (C) Western blot probed with anti-NIpl and anti-lbpA antibody showed that over-expression of $n / p /$ or $n / p /-M$ induced IbpA and/or IbpB in CP. (D)

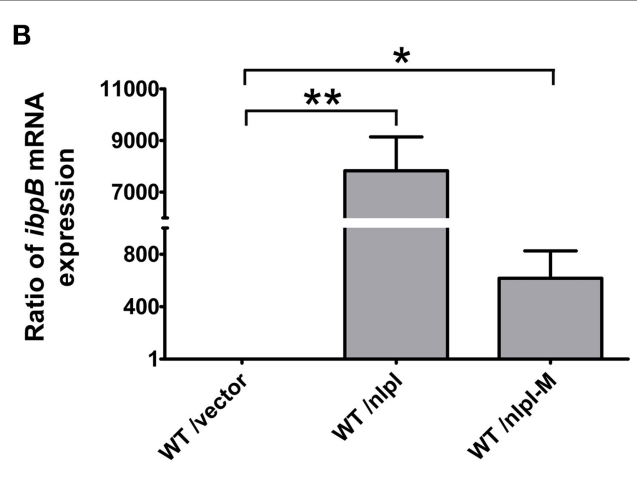

D

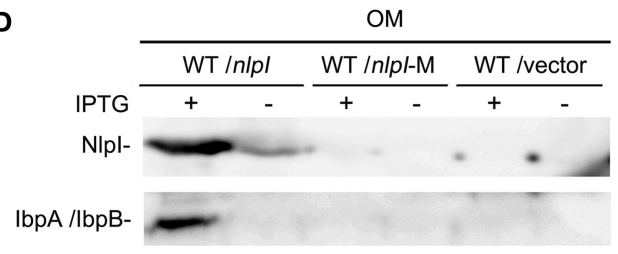

Western blot with anti-HisTag and anti-IbpA antibody showed that NIpl and IbpA/lbpB were present in OM of MG1655/pQE80-n/p/ with inducer, but did not detected NIpl and IbpA/lbpB in OM of MG1655/pQE80-n/p/-M with inducer. (E) The CP protein profiling of MG1655/pQE80-n/p/, MG1655/pQE80-n/p/-M and MG1655/pQE80L with/without inducer. ${ }^{* *} P<0.01,{ }^{*} P<0.05$.
IbpB were significantly increased in $\mathrm{CP}$ and $\mathrm{OM}$. The deletion of $i b p A$ or $i b p B$ and the trans-complementation of $\triangle i b p A$ experiments indicated that both $\mathrm{IbpA}$ and $\mathrm{IbpB}$ were involved in the NlpI-participated cell division.

Although the increase of NlpI protein level induced the expression of $i b p A$ and $i b p B$ and disrupted the bacterial nucleoids division, over-expression of $i b p A$ and/or $i b p B$ did not show similar phenotypes. In $\triangle i b p A, \triangle i b p B$ or $\triangle i b p A B$, nucleoids division was normal under the over-expression of $n l p I$, but the nucleoids were anomalous in the trans-complementation strains of $\triangle i b p A$ with the over-expression of $n l p I$ (Supplementary Figure S2D). The results indicated that inhibition nucleoids division of NlpI was dependent on the heat shock proteins IbpA and IbpB. Overexpression of $n l p I-M$ did not inhibit the bacterial growth and nucleoid division, but induced the mRNA levels of $i b p A$ and $i b p B$.
Moreover, the over-expression of nlpI-M caused the increase of IbpA/IbpB in CP, but not in OM. These results demonstrated that localization of IbpA/IbpB in OM played an important role in the NlpI-participated cell division. Co-immunoprecipitation assay, proteins MS analysis (Supplementary Figure S4 and Table S5) and bacterial two-hybrid assay showed that NlpI physically interacts with IbpB. Moreover, IbpA was not found to interact with NlpI, which highly suggests that the interaction between IbpB and NlpI is specific. It has been shown that IbpA interacts with IbpB (Matuszewska et al., 2005). We speculated that localization of NlpI in OM was essential for the export of IbpB, together with IbpA to OM and required for the NlpI-participated cell division. IbpA and IbpB play important roles in protecting recombinant proteins from degradation by cytoplasmic proteases (Han et al., 2004) and optimize recombinant proteins de novo 
A

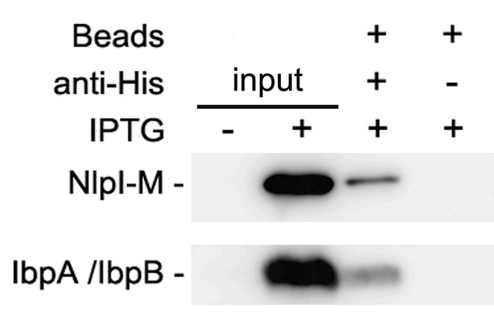

B
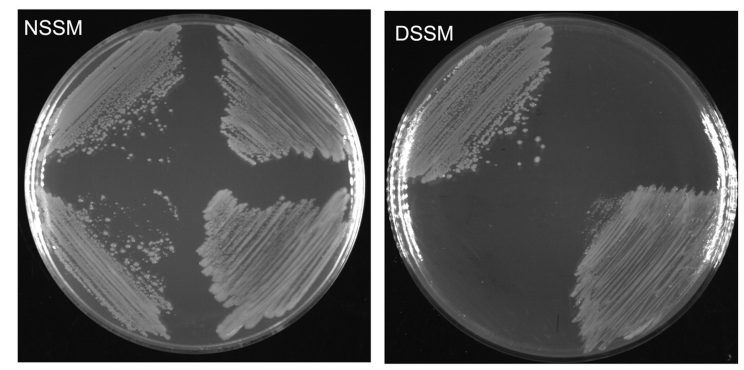

\begin{tabular}{l|l} 
pBT+ & pBT \\
pTRG+ & pTRG-ibpB \\
\hline pBT-nlpl & pBT-nlpl \\
pTRG & pTRG-ibpB
\end{tabular}

C
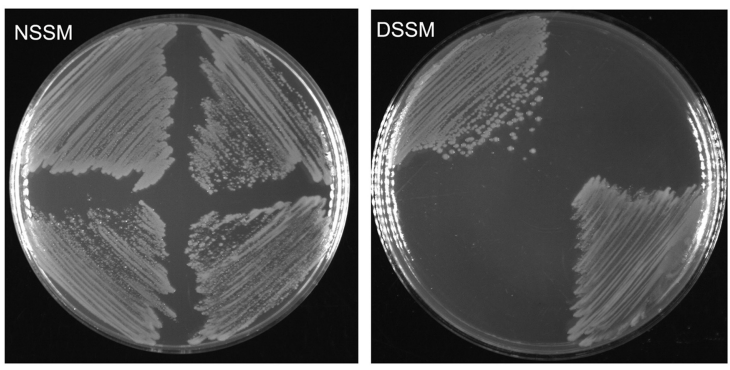

\begin{tabular}{l|l} 
pBT+ & pBT \\
pTRG+ & pTRG-ibpB \\
\hline pBT-nlpl-M & pBT-nlpl-M \\
pTRG & pTRG-ibpB
\end{tabular}

FIGURE 6 | NIpl physically interacts with IbpB. (A) Co-IP assays for the binding of NIpl-M with protein A beads by using anti-HisTag antibody. Immune complexes in MG1655/pQE80-n/p/-M induced with IPTG were collected, and the beads were washed with IP Lysis/Wash buffer. The samples were analyzed by using anti-IbpA antibody and anti-HisTag antibody, respectively. Lane 1 and lane 2 represented input CP of the bacteria without or with IPTG for Co-IP assay. Lane 3 and lane 4 are results of Co-IP assay. (B, C) Bacterial two-hybrid assays detected the interaction between IbpB and NIpl (B) or NIpl-M (C). NSSM, Non-selective Screening Medium. DSSM, Dual Selective Screening Media (plate contained str and $5 \mathrm{mM}$ 3-AT). $\mathrm{pBT}^{+}$and $\mathrm{pTRG}^{+}$, co-transformant containing pBT-LGF2 and PTRG-Gal11P as a positive control. folding (de Marco et al., 2007). Here, we found that IbpAB are esstential mediators in NlpI-participated cell division process. This is different from their above mentioned chaperones roles. We propose that IbpA, IbpB and NlpI form a protein complex, which plays an essential role in the detrimental effect of NlpI overexpression because deletion of IbpA or IbpB abolished the effect. This unkown protein complex will be explored in the future to find out the role of IbpAB in the NlpI-participated cell division.

The previous studies reported that the insertion mutant of $n l p I$ shows osmotic-sensitive growth at $42^{\circ} \mathrm{C}$ in E. coli (Ohara et al., 1999), and $n l p I$ is up-regulated in response to high-pressure and the deletion mutant of $n l p I$ is more sensitive to high-pressure than the wild type strain (Malone et al., 2006; Charoenwong et al., 2011). In this study, we found that the growth of $\Delta n l p I$ was inhibited more severely than the wild type strain at $50^{\circ} \mathrm{C}$ (Figure S5). Moreover, NlpI contributes to the cold acclimatization response in Salmonella Thyphimurium (Rouf et al., 2011b). We speculate that NlpI is a stress response protein and especially responds to high temperature. The up-regulation of $i b p A$ and $i b p B$ may be linked to the NlpI-participated stress response.

In summary, we found that over-expression of $n l p I$ interrupts the nucleoids division and the assembly of FtsZ at the septum, and $\mathrm{IbpA} / \mathrm{B}$ are required for this process, possibly by forming a $\mathrm{NlpI} / \mathrm{IbpA} / \mathrm{IbpB}$ protein complex. Other unknown factor(s) must be involved in the cell division defect caused by over-expression of $n l p I$. Since NlpI is a potential stress response protein, we proposed that NlpI can slow down the cell growth by inhibiting cell division to contribute the host survival when bacteria encounter heat shock stress.

\section{ACKNOWLEDGMENTS}

We gratefully thank Dr. Baoli Zhu and Dr. Yuanlong Pan at Institute of Microbiology, Chinese Academy of Sciences for the assistance in the microarray data analysis and Dr. Benfang Lei at Montana State University for helpful discussions and critical comments on the manuscript. This work 
was supported by grants from the National Natural Science Foundation of China (Number. 30870100, Number. 31270173), the State Key Development Programs for Basic Research of China (973 Program No. 2009CB522600 and 2015CB554203), and the Program for Professor of Special Appointment (Eastern Scholar) at Shanghai Institutions of Higher Learning.

\section{SUPPLEMENTARY MATERIAL}

The Supplementary Material for this article can be found online at: http://www.frontiersin.org/journal/10.3389/fmicb. 2015.00051/abstract

\section{REFERENCES}

Allen, S. P., Polazzi, J. O., Gierse, J. K., and Easton, A. M. (1992). Two novel heat shock genes encoding proteins produced in response to heterologous protein expression in Escherichia coli. J. Bacteriol. 174, 6938-6947.

Barnich, N., Bringer, M. A., Claret, L., and Darfeuille-Michaud, A. (2004). Involvement of lipoprotein NlpI in the virulence of adherent invasive Escherichia coli strain LF82 isolated from a patient with Crohn's disease. Infect. Immun. 72, 2484-2493. doi: 10.1128/IAI.72.5.2484-2493.2004

Bi, E. F., and Lutkenhaus, J. (1991). FtsZ ring structure associated with division in Escherichia coli. Nature 354, 161-164. doi: 10.1038/354161a0

Bi, E., and Lutkenhaus, J. (1993). Cell division inhibitors SulA and MinCD prevent formation of the FtsZ ring. J. Bacteriol. 175, 1118-1125.

Charoenwong, D., Andrews, S., and Mackey, B. (2011). Role of rpoS in the development of cell envelope resilience and pressure resistance in stationary-phase Escherichia coli. Appl. Environ. Microbiol. 77, 5220-5229. doi: 10.1128/AEM.00648-11

Crago, A. M., and Koronakis, V. (1998). Salmonella InvG forms a ring-like multimer that requires the InvH lipoprotein for outer membrane localization. Mol. Microbiol. 30, 47-56. doi: 10.1046/j.1365-2958.1998.01036.x

Das, A. K., Cohen, P. W., and Barford, D. (1998). The structure of the tetratricopeptide repeats of protein phosphatase 5: implications for TPR-mediated proteinprotein interactions. EMBO J. 17, 1192-1199. doi: 10.1093/emboj/17.5.1192

Datsenko, K. A., and Wanner, B. L. (2000). One-step inactivation of chromosomal genes in Escherichia coli K-12 using PCR products. Proc. Natl. Acad. Sci. U.S.A. 97, 6640-6645. doi: 10.1073/pnas.120163297

de Marco, A., Deuerling, E., Mogk, A., Tomoyasu, T., and Bukau, B. (2007). Chaperone-based procedure to increase yields of soluble recombinant proteins produced in E. coli. BMC Biotechnol. 7:32. doi: 10.1186/1472-6750-7-32

Du, Y., Zhang, H., He, Y., Huang, F., and He, Z. G. (2012). Mycobacterium smegmatis Lsr2 physically and functionally interacts with a new flavoprotein involved in bacterial resistance to oxidative stress. J. Biochem. 152, 479-486. doi: 10.1093/jb/mvs095

Egan, A. J., Jean, N. L., Koumoutsi, A., Bougault, C. M., Biboy, J., Sassine, J., et al. (2014). Outer-membrane lipoprotein LpoB spans the periplasm to stimulate the peptidoglycan synthase PBP1B. Proc. Natl. Acad. Sci. U.S.A. 111, 8197-8202. doi: 10.1073/pnas.1400376111

Ehlert, K., Holtje, J. V., and Templin, M. F. (1995). Cloning and expression of a murein hydrolase lipoprotein from Escherichia coli. Mol. Microbiol. 16, 761-768. doi: 10.1111/j.1365-2958.1995.tb02437.x

Gaubig, L. C., Waldminghaus, T., and Narberhaus, F. (2011). Multiple layers of control govern expression of the Escherichia coli ibpAB heat-shock operon. Microbiology 157, 66-76. doi: 10.1099/mic.0.043802-0

Gill, R. T., Valdes, J. J., and Bentley, W. E. (2000). A comparative study of global stress gene regulation in response to overexpression of recombinant proteins in Escherichia coli. Metab. Eng. 2, 178-189. doi: 10.1006/mben.2000.0148

Han, M. J., Park, S. J., Park, T. J., and Lee, S. Y. (2004). Roles and applications of small heat shock proteins in the production of recombinant proteins in Escherichia coli. Biotechnol. Bioeng. 88, 426-436. doi: 10.1002/bit.20227

Hiraga, S., Niki, H., Ogura, T., Ichinose, C., Mori, H., Ezaki, B., et al. (1989). Chromosome partitioning in Escherichia coli: novel mutants producing anucleate cells. J. Bacteriol. 171, 1496-1505.

Ichihara, S., Hussain, M., and Mizushima, S. (1981). Characterization of new membrane lipoproteins and their precursors of Escherichia coli. J. Biol. Chem. 256, 3125-3129.
Jiao, W., Qian, M., Li, P., Zhao, L., and Chang, Z. (2005). The essential role of the flexible termini in the temperature-responsiveness of the oligomeric state and chaperone-like activity for the polydisperse small heat shock protein IbpB from Escherichia coli. J. Mol. Biol. 347, 871-884. doi: 10.1016/j.jmb.2005.01.029

Jurgen, B., Lin, H. Y., Riemschneider, S., Scharf, C., Neubauer, P., Schmid, R., et al. (2000). Monitoring of genes that respond to overproduction of an insoluble recombinant protein in Escherichia coli glucose-limited fedbatch fermentations. Biotechnol. Bioeng. 70, 217-224. doi: 10.1002/10970290(20001020)70:2<217::AID-BIT11>3.0.CO;2-W

Kitagawa, M., Matsumura, Y., and Tsuchido, T. (2000). Small heat shock proteins, IbpA and IbpB, are involved in resistances to heat and superoxide stresses in Escherichia coli. FEMS Microbiol. Lett. 184, 165-171. doi: 10.1111/j.15746968.2000.tb09009.x

Kitagawa, M., Miyakawa, M., Matsumura, Y., and Tsuchido, T. (2002). Escherichia coli small heat shock proteins, IbpA and IbpB, protect enzymes from inactivation by heat and oxidants. Eur. J. Biochem. 269, 2907-2917. doi: 10.1046/j.14321033.2002.02958.x

Kuczynska-Wisnik, D., Laskowska, E., and Taylor, A. (2001). Transcription of the ibpB heat-shock gene is under control of sigma(32)- and sigma(54)-promoters, a third regulon of heat-shock response. Biochem. Biophys. Res. Commun. 284, 57-64. doi: 10.1006/bbrc.2001.4926

Kuczynska-Wisnik, D., Zurawa-Janicka, D., Narkiewicz, J., Kwiatkowska, J., Lipinska, B., and Laskowska, E. (2004). Escherichia coli small heat shock proteins IbpA/B enhance activity of enzymes sequestered in inclusion bodies. Acta Biochim. Pol. 51, 925-931.

Laskowska, E., Wawrzynow, A., and Taylor, A. (1996). IbpA and IbpB, the new heat-shock proteins, bind to endogenous Escherichia coli proteins aggregated intracellularly by heat shock. Biochimie 78, 117-122. doi: 10.1016/03009084(96)82643-5

Li, Y., Hsin, J., Zhao, L., Cheng, Y., Shang, W., Huang, K. C., et al. (2013). FtsZ protofilaments use a hinge-opening mechanism for constrictive force generation. Science 341, 392-395. doi: 10.1126/science. 1239248

Ma, X., Ehrhardt, D. W., and Margolin, W. (1996). Colocalization of cell division proteins FtsZ and FtsA to cytoskeletal structures in living Escherichia coli cells by using green fluorescent protein. Proc. Natl. Acad. Sci. U.S.A. 93, 12998-13003. doi: 10.1073/pnas.93.23.12998

Malone, A. S., Chung, Y. K., and Yousef, A. E. (2006). Genes of Escherichia coli O157:H7 that are involved in high-pressure resistance. Appl. Environ. Microbiol. 72, 2661-2671. doi: 10.1128/AEM.72.4.2661-2671.2006

Matuszewska, M., Kuczynska-Wisnik, D., Laskowska, E., and Liberek, K. (2005). The small heat shock protein IbpA of Escherichia coli cooperates with IbpB in stabilization of thermally aggregated proteins in a disaggregation competent state. J. Biol. Chem. 280, 12292-12298. doi: 10.1074/jbc.M412706200

Mogk, A., Mayer, M. P., and Deuerling, E. (2002). Mechanisms of protein folding: molecular chaperones and their application in biotechnology. Chembiochem 3, 807-814. doi: 10.1002/1439-7633(20020902)3:9<807::AIDCBIC807>3.0.CO;2-A

Mukherjee, A., Cao, C., and Lutkenhaus, J. (1998). Inhibition of FtsZ polymerization by SulA, an inhibitor of septation in Escherichia coli. Proc. Natl. Acad. Sci. U.S.A. 95, 2885-2890. doi: 10.1073/pnas.95.6.2885

Nesta, B., Valeri, M., Spagnuolo, A., Rosini, R., Mora, M., Donato, P., et al. (2014). SslE elicits functional antibodies that impair in vitro mucinase activity and in vivo colonization by both intestinal and extraintestinal Escherichia coli strains. PLoS Pathog. 10:e1004124. doi: 10.1371/journal.ppat.1004124

Ohara, M., Wu, H. C., Sankaran, K., and Rick, P. D. (1999). Identification and characterization of a new lipoprotein, NlpI, in Escherichia coli K-12. J. Bacteriol. 181, 4318-4325.

Ratajczak, E., Zietkiewicz, S., and Liberek, K. (2009). Distinct activities of Escherichia coli small heat shock proteins IbpA and IbpB promote efficient protein disaggregation. J. Mol. Biol. 386, 178-189. doi: 10.1016/j.jmb.2008.12.009

Rouf, S. F., Ahmad, I., Anwar, N., Vodnala, S. K., Kader, A., Romling, U., et al. (2011a). Opposing contributions of polynucleotide phosphorylase and the membrane protein NlpI to biofilm formation by Salmonella enterica serovar Typhimurium. J. Bacteriol. 193, 580-582. doi: 10.1128/JB.00905-10

Rouf, S. F., Anwar, N., Clements, M. O., and Rhen, M. (2011b). Genetic analysis of the pnp-deaD genetic region reveals membrane lipoprotein NlpI as an independent participant in cold acclimatization of Salmonella enterica serovar Typhimurium. FEMS Microbiol. Lett. 325, 56-63. doi: 10.1111/j.15746968.2011.02416.x 
Sanchez-Torres, V., Maeda, T., and Wood, T. K. (2010). Global regulator H-NS and lipoprotein NlpI influence production of extracellular DNA in Escherichia coli. Biochem. Biophys. Res. Commun. 401, 197-202. doi: 10.1016/j.bbrc.2010. 09.026

Sorensen, H. P., and Mortensen, K. K. (2005). Advanced genetic strategies for recombinant protein expression in Escherichia coli. J. Biotechnol. 115, 113-128. doi: 10.1016/j.jbiotec.2004.08.004

Strozecka, J., Chrusciel, E., Gorna, E., Szymanska, A., Zietkiewicz, S., and Liberek, K. (2012). Importance of $\mathrm{N}$ - and C-terminal regions of IbpA, Escherichia coli small heat shock protein, for chaperone function and oligomerization. J. Biol. Chem. 287, 2843-2853. doi: 10.1074/jbc.M111. 273847

Tadokoro, A., Hayashi, H., Kishimoto, T., Makino, Y., Fujisaki, S., and Nishimura, Y. (2004). Interaction of the Escherichia coli lipoprotein NlpI with periplasmic Prc (Tsp) protease. J. Biochem. 135, 185-191. doi: 10.1093/jb/ mvh022

Teng, C. H., Tseng, Y. T., Maruvada, R., Pearce, D., Xie, Y., Paul-Satyaseela, M., et al. (2010). NlpI contributes to Escherichia coli K1 strain RS218 interaction with human brain microvascular endothelial cells. Infect. Immun. 78, 3090-3096. doi: 10.1128/IAI.00034-10

Tseng, Y. T., Wang, S. W., Kim, K. S., Wang, Y. H., Yao, Y., Chen, C. C., et al. (2012). NlpI facilitates deposition of C4bp on Escherichia coli by blocking classical complement-mediated killing, which results in high-level bacteremia. Infect. Immun. 80, 3669-3678. doi: 10.1128/IAI.00320-12

Wai, S. N., Westermark, M., Oscarsson, J., Jass, J., Maier, E., Benz, R., et al. (2003). Characterization of dominantly negative mutant ClyA cytotoxin proteins in Escherichia coli. J. Bacteriol. 185, 5491-5499. doi: 10.1128/JB.185.18.54915499.2003

Wilson, C. G., Kajander, T., and Regan, L. (2005). The crystal structure of NlpI. A prokaryotic tetratricopeptide repeat protein with a globular fold. FEBS J. 272, 166-179. doi: 10.1111/j.1432-1033.2004.04397.x

Wu, L. J., and Errington, J. (2012). Nucleoid occlusion and bacterial cell division. Nat. Rev. Microbiol. 10, 8-12. doi: 10.1038/nrmicro2671

Yao, Y., Sturdevant, D. E., and Otto, M. (2005). Genomewide analysis of gene expression in Staphylococcus epidermidis biofilms: insights into the pathophysiology of S. epidermidis biofilms and the role of phenol-soluble modulins in formation of biofilms. J. Infect. Dis. 191, 289-298. doi: 10.1086/426945 Yao, Y., Xie, Y., and Kim, K. S. (2006). Genomic comparison of Escherichia coli K1 strains isolated from the cerebrospinal fluid of patients with meningitis. Infect. Immun. 74, 2196-2206. doi: 10.1128/IAI.74.4.2196-2206.2006

Yu, F., Inouye, S., and Inouye, M. (1986). Lipoprotein-28, a cytoplasmic membrane lipoprotein from Escherichia coli. Cloning, DNA sequence, and expression of its gene. J. Biol. Chem. 261, 2284-2288.

Zhou, Y., Tao, J., Yu, H., Ni, J., Zeng, L., Teng, Q., et al. (2012). Hcp family proteins secreted via the type VI secretion system coordinately regulate Escherichia coli $\mathrm{K} 1$ interaction with human brain microvascular endothelial cells. Infect. Immun. 80, 1243-1251. doi: 10.1128/IAI.05994-11

Zuckert, W. R. (2014). Secretion of bacterial lipoproteins: through the cytoplasmic membrane, the periplasm and beyond. Biochim. Biophys. Acta 1843, 1509-1516. doi: 10.1016/j.bbamcr.2014.04.022

Conflict of Interest Statement: The Review Editor Ching-Hao Teng declares that, despite having collaborated with author Yufeng Yao, the review process was handled objectively and no conflict of interest exists. The authors declare that the research was conducted in the absence of any commercial or financial relationships that could be construed as a potential conflict of interest.

Received: 02 September 2014; accepted: 15 January 2015; published online: 04 February 2015.

Citation: Tao J, Sang Y, Teng Q, Ni J, Yang Y, Tsui SK-W and Yao Y-F (2015) Heat shock proteins IbpA and IbpB are required for NlpI-participated cell division in Escherichia coli. Front. Microbiol. 6:51. doi: 10.3389/fmicb.2015.00051

This article was submitted to Food Microbiology, a section of the journal Frontiers in Microbiology.

Copyright (c) 2015 Tao, Sang, Teng, Ni, Yang, Tsui and Yao. This is an open-access article distributed under the terms of the Creative Commons Attribution License (CC BY). The use, distribution or reproduction in other forums is permitted, provided the original author(s) or licensor are credited and that the original publication in this journal is cited, in accordance with accepted academic practice. No use, distribution or reproduction is permitted which does not comply with these terms. 